\title{
Effects of hydrogenation on non-radiative defects in GaNP and GaNAsalloys: An optically detected magnetic resonance study
}

Daniel Dagnelund, I.P. Vorona, G. Nosenko, X. J. Wang, C. W. Tu, H. Yonezu, A. Polimeni, M. Capizzi, Weimin Chen and Irina Buyanova

\section{Linköping University Post Print}

N.B.: When citing this work, cite the original article.

Original Publication:

Daniel Dagnelund, I.P. Vorona, G. Nosenko, X. J. Wang, C. W. Tu, H. Yonezu, A. Polimeni, M. Capizzi, Weimin Chen and Irina Buyanova, Effects of hydrogenation on non-radiative defects in GaNP and GaNAsalloys: An optically detected magnetic resonance study, 2012, Journal of Applied Physics, (111), 023501, . http://dx.doi.org/10.1063/1.3676576

Copyright: American Institute of Physics (AIP) http://www.aip.org/

Postprint available at: Linköping University Electronic Press http://urn.kb.se/resolve?urn=urn:nbn:se:liu:diva-74675 


\title{
Effects of hydrogenation on non-radiative defects in GaNP and GaNAs alloys: An optically detected magnetic resonance study
}

\author{
D. Dagnelund, ${ }^{1}$ I. P. Vorona, ${ }^{1,2}$ G. Nosenko, ${ }^{1,2}$ X. J. Wang,,${ }^{1,3}$ C. W. Tu, ${ }^{4}$ H. Yonezu, ${ }^{5}$ \\ A. Polimeni, ${ }^{6}$ M. Capizzi, ${ }^{6}$ W. M. Chen, ${ }^{1}$ and I. A Buyanova ${ }^{1, a)}$ \\ ${ }^{1}$ Department of Physics, Chemistry and Biology, Linköping University, S-581 83 Linköping, Sweden \\ ${ }^{2}$ Institute of Semiconductor Physics, National Academy of Sciences of Ukraine, Kiev 03028, Ukraine \\ ${ }^{3}$ National Laboratory for Infrared Physics, Shanghai Institute of Technical Physics, \\ Chinese Academy of Sciences, 200083 Shanghai, China \\ ${ }^{4}$ Department of Electrical and Computer Engineering, University of California, San Diego, La Jolla, \\ California 92093, USA \\ ${ }^{5}$ Department of Electrical and Electronic Engineering, Toyohashi University of Technology, Toyohashi, \\ Aichi, 441-8580, Japan \\ ${ }^{6}$ INFM and Dipartimento di Fisica, Università di Roma "La Sapienza", Piazzale A. Moro 2, \\ I-00185 Roma, Italy
}

(Received 5 October 2011; accepted 14 December 2011; published online 17 January 2012)

Photoluminescence and optically detected magnetic resonance techniques are utilized to study defect properties of GaNP and GaNAs alloys subjected to post-growth hydrogenation by low-energy sub-threshold ion beam irradiation. It is found that in GaNP $\mathrm{H}$ incorporation leads to activation of new defects, which has a $\mathrm{Ga}$ interstitial $\left(\mathrm{Ga}_{\mathrm{i}}\right)$ atom at its core and may also involve a $\mathrm{H}$ atom as a partner. The observed activation critically depends on the presence of $\mathrm{N}$ in the alloy, as it does not occur in GaP with a low level of $\mathrm{N}$ doping. In sharp contrast, in GaNAs hydrogen is found to efficiently passivate $\mathrm{Ga}_{\mathrm{i}}$-related defects present in the as-grown material. A possible mechanism responsible for the observed difference in the $\mathrm{H}$ behavior in GaNP and GaNAs is discussed. (C) 2012 American Institute of Physics. [doi:10.1063/1.3676576]

\section{INTRODUCTION}

Hydrogen is one of the most common impurities in semiconductors. It is abundant in many steps of semiconductor growth and device processing. Due to its high chemical reactivity, hydrogen is known to efficiently interact with nearly all types of imperfections and impurities present in semiconductors, which most often leads to their passivation. ${ }^{1,2}$ Well known examples of impurities/defects that can be efficiently passivated by hydrogen include $\mathrm{B}$ acceptors ${ }^{3,4}$ and $\mathrm{P}$ donors ${ }^{5}$ in $\mathrm{Si}$, the $\mathrm{V}$-O centers in $\mathrm{Si}$ and $\mathrm{SiGe} / \mathrm{Si}$ heterostructures,,${ }^{6,7} \mathrm{Be}$ acceptors in $\mathrm{AlGaAs} / \mathrm{GaAs}$ quantum wells $(\mathrm{QW}),{ }^{8}$ as well as silicon dangling bonds $\left(\mathrm{P}_{\mathrm{b}}\right.$ defect) at a $\mathrm{Si} / \mathrm{SiO}_{2}$ interface. ${ }^{9}$ In fact, the passivation of the $\mathrm{P}_{\mathrm{b}}$ defects is absolutely essential for reliable operation of metal-oxide-semiconductor fieldeffect-transistors, the heart of integrated circuit technology. In some rare cases, $\mathrm{H}$ was also found to activate defects and impurities such as Ga vacancy in GaAs (Ref. 10) and neutral dopants, such as Si and C in ultrapure Ge. ${ }^{11}$

Hydrogen is also known to greatly affect properties of dilute nitrides, such as $\mathrm{Ga}(\mathrm{In}) \mathrm{NAs}$ and GaNP alloys, which are novel materials promising for a variety of applications in, e.g., laser diodes for fiber-optic communications, highly efficient visible light emitting diodes, multi-junction solar cells, as well as in III-V optoelectronic integrated circuits on Si wafers. ${ }^{12}$ Most unexpectedly in these materials, incorporation of $\mathrm{H}$ was found to cause dramatic changes in the fundamental band structure by effectively neutralizing all alloy properties caused by the presence of nitrogen and recovering the band-gap energy, electron effective mass and other

${ }^{a)}$ Electronic mail: irb@ifm.liu.se. parameters of the N-free hosts. ${ }^{13-18}$ Though this unusual $\mathrm{H}$ behavior has attracted a great deal of attention, studies devoted to effects of hydrogenation on grown-in defects in dilute nitrides remain scarce. This is in spite of the fact that defect formation leading to efficient non-radiative recombination (NRR) is known to be very severe in dilute nitrides ${ }^{19}$ and in fact presents one of the key obstacles for widespread applications of these materials. For example, Wang et al. have recently shown ${ }^{20}$ that up to $88 \%$ of recombination in $\mathrm{Ga}(\mathrm{In}) \mathrm{NAs}$ alloys suffers non-radiative losses via NRR and have identified a complex involving a $\mathrm{Ga}$ interstitial $\left(\mathrm{Ga}_{\mathrm{i}}\right)$ as the responsible defect. Moreover, growth processes utilized for fabrication of dilute nitrides, such as gas-source molecular beam epitaxy (GS-MBE) or metalorganic chemical-vapor deposition (MOCVD), usually involve hydrogen. ${ }^{21,22}$ Since $\mathrm{H}$ is a common contaminant in dilute nitrides and may affect defect formation, ${ }^{23}$ a better understanding and control of the grown-in defects and of their interaction with hydrogen in dilute nitrides is highly desirable. Most recently, we have addressed ${ }^{24}$ this issue by investigating the effects that low energy sub-threshold H-treatment has on defect properties of GaNP alloys with a relatively low $\mathrm{N}$ content of $0.6 \%-0.8 \%$. An unexpected activation of several defects with a $\mathrm{Ga}_{\mathrm{i}}$ atom at the core has been found, which provides an explanation for the puzzling observation of deterioration of material quality after hydrogenation. Since neither of these $\mathrm{Ga}_{\mathrm{i}}$-related defects has previously been detected in GaNP, they were tentatively suggested to involve $\mathrm{H}$ atoms. In this work we extend these studies to GaNP alloys with different $\mathrm{N}$ contents and concentrations of grown-in defects present before the $\mathrm{H}$ treatment and also to GaNAs alloys, aiming to reveal 
common trends in defect formation. Photoluminescence (PL) and optically detected magnetic resonance techniques (ODMR) will be employed for these purposes.

\section{SAMPLES AND METHODS}

Two sets of GaNP/GaP and GaNAs/GaAs structures grown by gas-source molecular beam epitaxy were chosen for this study. Each set contains three structures with distinctly different $\mathrm{N}$ compositions and, therefore, different concentrations/types of grown-in defects active in recombination prior to the $\mathrm{H}$ treatment. The most important growth parameters of the structures are summarized in Table I. Post-growth hydrogenation was performed by ion-beam irradiation from a Kaufmann source at $300^{\circ} \mathrm{C}$, using low ion energy $(100 \mathrm{eV})$ and current density of $\sim 10 \mu \mathrm{A} / \mathrm{cm}^{2}$. Samples were hydrogenated with the same dose of $[\mathrm{H}]=1 \times 10^{18} \mathrm{~cm}^{-2}$ except for the GaNAs/GaAs multiple quantum well (MQW) structure that was hydrogenated with a $\mathrm{H}$ dose of $4.5 \times 10^{18} \mathrm{~cm}^{-2}$.

Secondary ion mass spectrometry (SIMS) was used to confirm $\mathrm{H}$ incorporation and to determine distributions of $\mathrm{H}$ and $\mathrm{N}$ within several representative structures. Ion-implanted standards were used for calibration of the $\mathrm{H}$ and $\mathrm{N}$ concentrations to an accuracy of $\pm 20 \%$. Results of SIMS measurements for the investigated $\mathrm{GaNP} / \mathrm{GaP}$ and GaNAs/GaAs structures are summarized in Figs. 1 and 2, respectively. Depth profiles of $\mathrm{N}$ distributions from the as-grown samples confirm the intended sample structures during the growth. For example, uniform distributions of $\mathrm{N}$ were found in GaNP (Fig. 1) and GaNAs (Fig. 2(a)) epilayers, whereas the $\mathrm{N}$ profile for the GaNAs MQW structure (Fig. 2(b)) clearly shows the formation of 7 periods of GaNAs QWs separated by GaAs barriers. One also notices that the GaNAs epilayer (MQW structure) is capped by a 200 - $\AA$-thick (500-£-thick) GaAs layer. The background $\mathrm{H}$ concentration in the structures is around $\sim 1 \times 10^{18} \mathrm{~cm}^{-3}$ close to the buffer layer and gradually increases toward the surface, which is not surprising as $\mathrm{H}$ is abundantly present during the GS-MBE growth. All $\mathrm{N}$ profiles remain identical in the hydrogenated samples proving that no out-diffusion of nitrogen occurred during the $\mathrm{H}$ treatment. On the other hand, the $\mathrm{H}$ distributions dramatically change after the hydrogenation and become fully correlated with the $\mathrm{N}$ profiles which is in excellent agreement with our previous results for GaNAs and GaNP alloys hydrogenated using $\mathrm{H}$ plasma. ${ }^{25}$ Such correlated distributions of $\mathrm{H}$ with a certain element/impurity is typical when

TABLE I. List of the GaNP and GaNAs samples studied in this work, with the main growth parameters and hydrogen dose in the post-growth hydrogenation. The numbers 5 and 20 in the parentheses refer to the thicknesses of the GaNAs and GaAs layers in the MQW structure.

\begin{tabular}{lcccccc}
\hline \hline & \multicolumn{3}{c}{ GaNP/GaP } & \multicolumn{3}{c}{ GaNAs/GaAs } \\
\hline Sample No. & $\# 2666$ & $\# 2671$ & $\#$ L012 & $\# 2661$ & $\# 2468$ & $\# 2522$ \\
Growth T $\left({ }^{\circ} \mathrm{C}\right)$ & 520 & 520 & 590 & 420 & 420 & 420 \\
{$[\mathrm{~N}](\%)$} & 0.05 & 0.80 & 1.4 & 0.7 & 1.3 & 1.6 \\
Structure & Epi & epi & epi & Epi & epi & MQW \\
Thickness $(\mathrm{nm})$ & 250 & 250 & 100 & 100 & 110 & $7 \times(5 / 20)$ \\
{$[\mathrm{H}]\left(10^{18} \mathrm{~cm}^{-2}\right)$} & 1 & 1 & 1 & 1 & 1 & 4.5 \\
\hline \hline
\end{tabular}

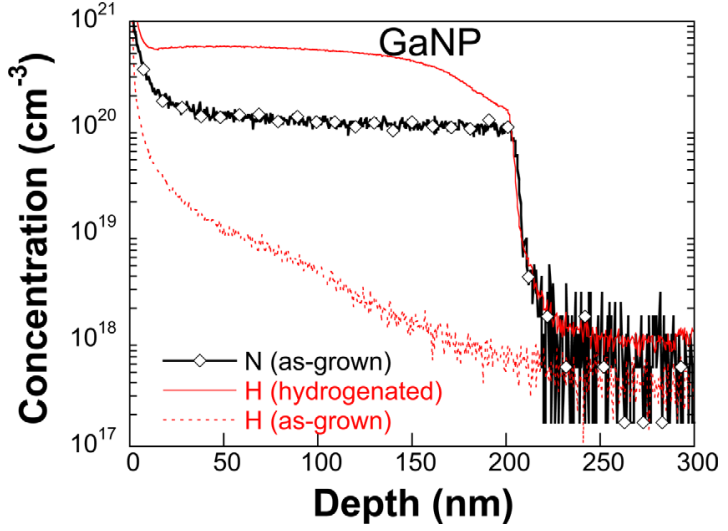

FIG. 1. (Color online) Representative SIMS profiles of $\mathrm{N}$ and $\mathrm{H}$ in the $\mathrm{GaN}_{0.008} \mathrm{P}_{0.992}$ epilayer before and after $\mathrm{H}$ treatment.

$\mathrm{H}$ passivates this impurity ${ }^{26}$ and, therefore, implies strong bonding of $\mathrm{H}$ to $\mathrm{N}$ atoms in our case. ${ }^{25,27,28}$

$\mathrm{PL}$ and ODMR measurements were performed at $5 \mathrm{~K}$ using as an excitation source the $532 \mathrm{~nm}(820-880 \mathrm{~nm})$ line of a solid state (Ti:Sapphire) laser in the case of GaNP (GaNAs). PL signals were dispersed by a $0.8 \mathrm{~m}$ double grating monochromator. A liquid nitrogen cooled Ge detector was used for detection in the near infrared (NIR) spectral range whereas visible $\mathrm{PL}$ was detected by a $\mathrm{Si}$ photodiode. ODMR signals were measured at X-band $(9.214 \mathrm{GHz})$ as spin-resonance induced changes of the PL intensity and were detected by the lock-in technique in phase with an amplitude modulated microwave field at a frequency of $3333 \mathrm{~Hz}$.

\section{RESULTS AND DISCUSSION}

\section{A. GaNP alloys}

Figure 3 summarizes the effects of post-growth hydrogenation on ODMR spectra of GaNP alloys with different $\mathrm{N}$ compositions. These spectra were monitored by measuring

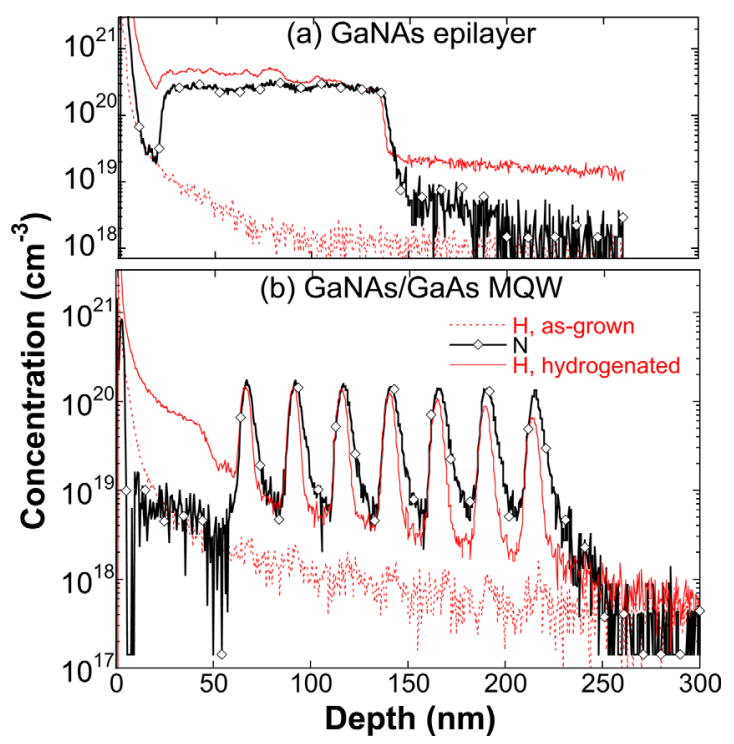

FIG. 2. (Color online) Representative SIMS profiles of $\mathrm{N}$ and $\mathrm{H}$ in the $\mathrm{GaN}_{0.013} \mathrm{As}_{0.987}$ epilayer (a) and $\mathrm{GaN}_{0.016} \mathrm{As}_{0.984} / \mathrm{GaAs} \mathrm{MQW}$ (b) before and after $\mathrm{H}$ treatment. 


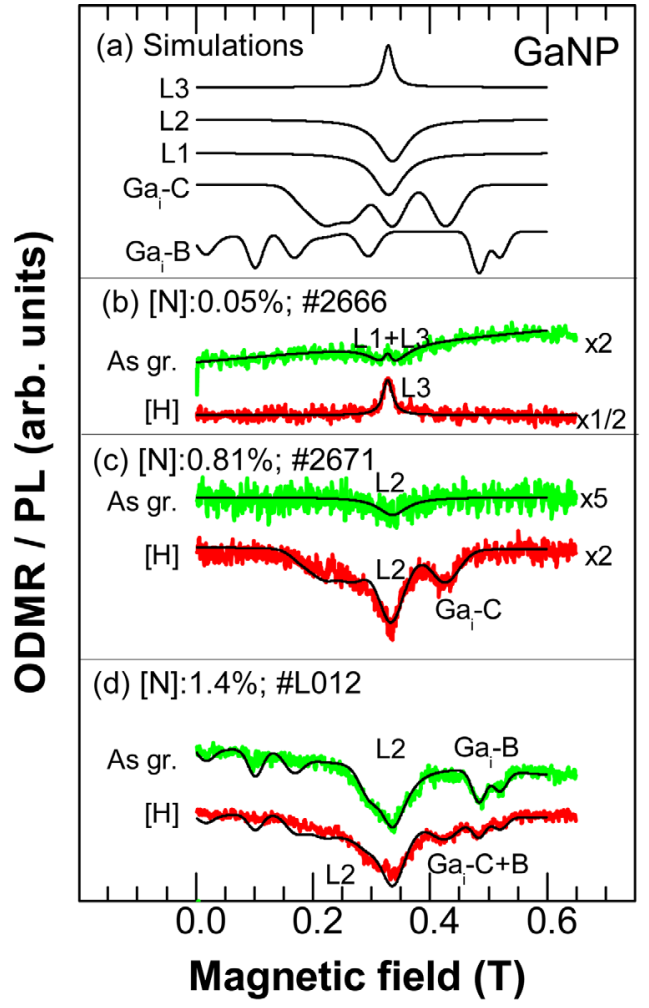

FIG. 3. (Color online) ODMR spectra from GaNP epilayers detected at $5 \mathrm{~K}$ by monitoring the PL emission within the visible $(550-810 \mathrm{~nm})$ spectral range. The ODMR signals are isotropic and are normalized to the PL intensity. Simulated ODMR signals are displayed in (a) whereas the experimentally measured spectra are shown in (b)-(d) for the specified $\mathrm{N}$ compositions. The simulated ODMR spectra are also shown in (b)-(d) by the thin black lines. The spin Hamiltonian parameters used in the simulated spectra are given in Table II.

near-band-edge PL emissions due to radiative recombination at $\mathrm{N}$-related localized states ${ }^{29}$ as shown in Fig. 4. A redshift of these emissions observed with increasing $\mathrm{N}$ content reflects a reduction of the bandgap energy of the alloys due to the bowing in the bandgap energy. On the other hand, a blueshift occurs after hydrogenation, which is due to a partial $\mathrm{H}$-induced recovery of the energy bandgap and can be viewed as a decrease in the effective $\mathrm{N}$ concentration. All these effects of $\mathrm{H}$ on the PL properties in GaNP are well documented in the literature ${ }^{12}$ and, therefore, will not be further discussed here. According to results shown in Fig. 3, post growth hydrogenation causes a dramatic increase in the intensity of the ODMR spectra in all investigated structures due to an appearance of new signals. Except for the ODMR line labeled as L3 in Fig. 3(b), all recorded ODMR signals are negative, i.e., they correspond to a decrease in the intensity of the monitored near-band-edge PL under the spin resonance conditions. This means that the spin-resonance enhanced recombination via the corresponding defects leads to a decrease in the PL intensity, i.e., that the defects act as competing recombination centers and degrade the optical quality of the alloy. ${ }^{30}$

The observed changes in the ODMR spectra provide an unambiguous proof that the post growth hydrogenation has a strong effect on the defect properties of the alloy. Before analyzing these effects, however, we would like to provide a

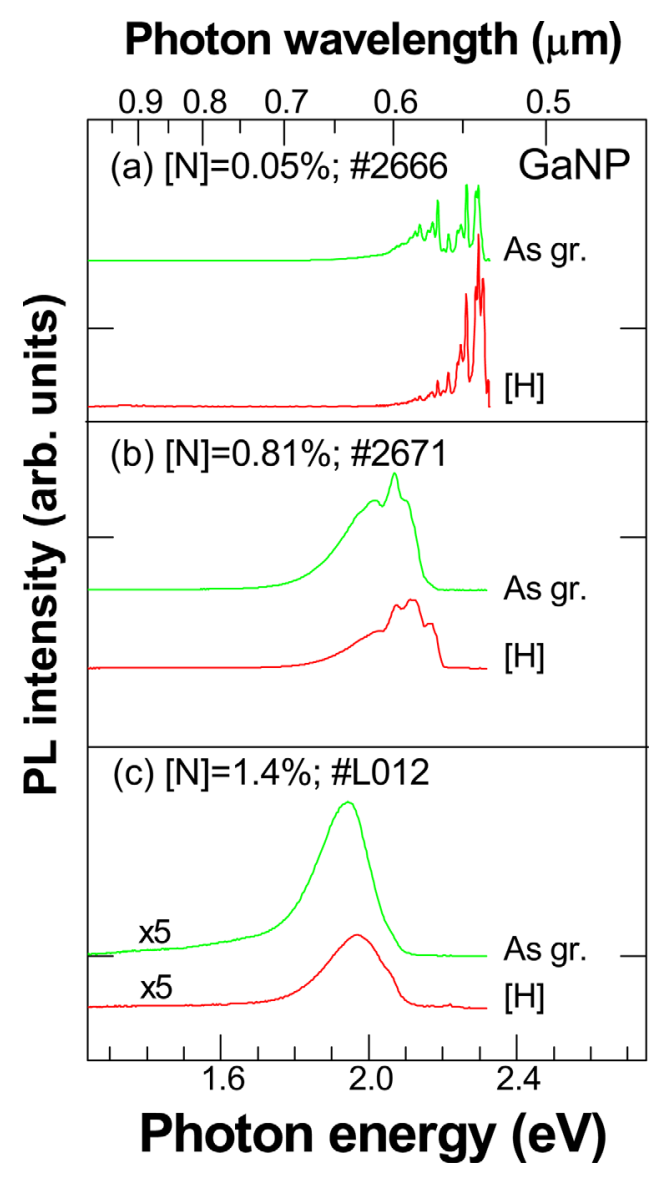

FIG. 4. (Color online) Typical PL spectra measured at $5 \mathrm{~K}$ from the GaNP epilayers with the $\mathrm{N}$ compositions of (a) $0.05 \%$, (b) $0.81 \%$, and (c) $1.4 \%$, before and after hydrogenation.

brief overview of the detected ODMR signals which can be attributed to several different paramagnetic centers. They are labeled as $\mathrm{Ga}_{i}-\mathrm{B}, \mathrm{Ga}_{i}-\mathrm{C}, \mathrm{L} 1-\mathrm{L} 3$ in Fig. 3. The first two signals contain a rich pattern of lines spreading over a wide field range, with the most prominent peaks in the region of $0.4-0.6 \mathrm{~T}$. On the other hand, the L1-L3 ODMR signals are merely single Lorentzian lines peaking at around $0.33 \mathrm{~T}$, each with a different linewidth. Spin Hamiltonian parameters of the related defects were obtained by analyzing the measured signals with the aid of a spin Hamiltonian that includes an electron Zeeman and central hyperfine interaction terms,

$$
H=\mu_{B} \mathbf{B} \cdot \mathbf{g} \cdot \mathbf{S}+\mathbf{S} \cdot \mathbf{A} \cdot \mathbf{I} .
$$

Here, $\mu_{B}$ is the Bohr magnetron, $\mathbf{B}$ is the magnetic field, $\mathbf{g}$ is the electronic g-tensor, and $\mathbf{A}$ is the central hyperfine tensor for each isotope. Since all observed ODMR signals are isotropic, $\mathbf{g}$ and $\mathbf{A}$ tensors are reduced to scalars $\mathrm{g}$ and $\mathrm{A}$. The electronic and nuclear spin of the studied defects are denoted by $\mathbf{S}$ and $\mathbf{I}$, respectively. It was found that the single lines L1 - L3 originate from paramagnetic centers with an effective electron spin $S=1 / 2$ and a $g$-factor close to 2 . Unfortunately, no chemical identification of the corresponding defects is possible from the current study, due to a lack of the resolved hyperfine structure. These defects, therefore, will not be further discussed in the paper. The signals $\mathrm{Ga}_{i}-\mathrm{B}$ 
and $\mathrm{Ga}_{i}-\mathrm{C}$, on the other hand, exhibit a well-resolved hyperfine structure arising from the interaction between the electron spin $S=1 / 2$ and the nuclear spin $I=3 / 2$ of an interstitial $\mathrm{Ga}_{i}$ atom in the core of the defect. ${ }^{31,32}$ Spin Hamiltonian parameters of all detected paramagnetic centers are given in Table II, whereas the corresponding simulated ODMR spectra are shown in Fig. 3(a). For all samples, the results of the simulations (shown by the black thin lines in Figs. 3(b)-3(d)), including contributions from the specified centers, are also overlaid with the experimentally measured ODMR spectra. The agreement between the simulations and experimental results is rather satisfactory, ${ }^{33}$ thus justifying the assignments of the defects and reliability of the obtained fitting parameters. As all defects observed in this study possess a unique set of the spin Hamiltonian parameters, the ODMR technique can be employed to individually monitor formation of these defects as a function of $\mathrm{N}$ and $\mathrm{H}$ content, as it will be shown in the following.

Let us now discuss how incorporation of $\mathrm{H}$ affects paramagnetic defects in the GaNP alloys. The most pronounced effect, which is common for all GaNP alloys, is the hydrogen-induced activation/creation of the $\mathrm{Ga}_{\mathrm{i}}-\mathrm{C}$ defect. This effect is most apparent in the alloys where no $\mathrm{Ga}_{\mathrm{i}}$-defects were detectable prior to the hydrogenation (e.g., with $[\mathrm{N}]=0.81 \%$ - see Fig. 3(c)), as has been reported in our earlier study. ${ }^{24}$ It also occurs in the alloys with a higher $\mathrm{N}$ content (e.g., $[\mathrm{N}]=1.4 \%$ ) where the $\mathrm{Ga}_{\mathrm{i}}$ defects were already formed in the as-grown material but in a different configuration, i.e., $\mathrm{Ga}_{\mathrm{i}}-\mathrm{B}$, see Fig. 3(d). Such defect activation by hydrogen is rather unusual and has only been reported in a handful of cases. We have previously argued that this activation cannot be attributed to defect creation caused by barely kick-out of a $\mathrm{Ga}$ atom by an $\mathrm{H}$ ion from a substitutional to interstitial site, as the energy that can be transferred from a $100 \mathrm{eV} \mathrm{H}$ ion to a $\mathrm{Ga}$ atom in a direct collision is only $5.6 \mathrm{eV}$, i.e., below the threshold displacement energy of about $8.8 \mathrm{eV}$ for $\mathrm{Ga}$ atoms. ${ }^{34}$ Our present results further support this conclusion. Indeed, even though the same kick-out process is also expected to occur in $\mathrm{GaNP}$ independent of $\mathrm{N}$ composition, the $\mathrm{Ga}_{\mathrm{i}} \mathrm{C}$ defects were not formed after hydrogenation in GaNP with a low $\mathrm{N}$ content as can be seen from Fig. 3(b). Therefore, the emergence of these defects must be due to their activation by hydrogen that could be accomplished via several mechanisms. First of all, it could be due to a hydrogen-induced change in the Fermi level position, which favors the paramagnetic charge state of $\mathrm{Ga}_{\mathrm{i}} \mathrm{C}$, i.e., detectable via spin resonance. However, this explanation seems to be somewhat less likely as we have so far never observed the $\mathrm{Ga}_{\mathrm{i}}-\mathrm{C}$ defect in GaNP alloys that were not irradiated by $\mathrm{H}$ atoms. This is in spite of the fact that the studied samples span over a wide range in $\mathrm{N}$ compositions, doping and also residual contamination. The second and more probable mechanism is formation of complexes between $\mathrm{H}$ atoms supplied by the hydrogenation and $\mathrm{a} \mathrm{Ga}_{\mathrm{i}}$ atom that was already present in an ODMR-inactive state in the as-grown sample. This might result either in a change in the position of the defect energy level or in its charge state toward the spin-active one, thereby activating the defects in carrier recombination monitored by ODMR. We can also conclude that the initial presence of $\mathrm{Ga}_{\mathrm{i}}$ should become energetically favorable only in materials containing a large amount of $\mathrm{N}$, as it does not occur in GaP: $\mathrm{N}$ with the low $\mathrm{N}$ composition of $[\mathrm{N}]=0.05 \%$, i.e., within the doping limit. However, the question whether the $\mathrm{N}$ atom is directly involved as a partner of the complex or barely promotes the formation of $\mathrm{Ga}_{\mathrm{i}}$ cannot be answered based on the currently available data, unfortunately, due to a lack of the resolved hyperfine structure related to $\mathrm{N}$.

We would like to note that $\mathrm{H}$ incorporation also causes a slight reduction of the ODMR signal from the $\mathrm{Ga}_{\mathrm{i}}-\mathrm{B}$ defect (see Fig. 3(d)). The observed minor effect in hydrogen passivation of defects that are active in recombination in the as-grown material is, however, somewhat surprising in view of the commonly known ability of $\mathrm{H}$ to passivate various deep centers in semiconductors.

\section{B. GaNAs alloys}

In order to evaluate to which extent the aforementioned $\mathrm{H}$-induced effects on defect properties are common for dilute nitrides, ODMR studies were extended to GaNAs alloys. To facilitate a direct comparison of results for both material systems, GaNAs epilayers were selected with similar N compositions as that in the case of GaNP.

The results of the performed ODMR measurements are summarized in Fig. 5, whereas Fig. 6 presents typical spectra of PL emissions used for detection of the ODMR signals. The near-band edge emission in GaNAs is due to recombination of excitons trapped by potential fluctuations of conduction band edge, ${ }^{35}$ whereas a broad PL band in the NIR spectral range is related to defects of unknown origin. Similar to the GaNP alloys, $\mathrm{H}$ incorporation leads to a blue shift

TABLE II. Spin Hamiltonian parameters and linewidth obtained from the best fit to the experimental ODMR results. The ratio $\mathrm{A}\left({ }^{71} \mathrm{Ga}\right) / \mathrm{A}\left({ }^{69} \mathrm{Ga}\right)$ was chosen as $1.27-1.3$, i.e., close to that of their nuclear magnetic moments $\mu(71 \mathrm{Ga}) / \mu(69 \mathrm{Ga})=1.27$.

\begin{tabular}{|c|c|c|c|c|c|c|c|c|c|}
\hline \multirow{2}{*}{$\frac{\text { Samples }}{\text { Defects }}$} & \multicolumn{5}{|c|}{$\mathrm{GaNP} / \mathrm{GaP}$} & \multicolumn{4}{|c|}{ GaNAs/GaAs } \\
\hline & L1 & L2 & L3 & $\mathrm{Ga}_{\mathrm{i}}-\mathrm{B}$ & $\mathrm{Ga}_{\mathrm{i}}-\mathrm{C}^{\mathrm{a}}$ & G1 & $\mathrm{Ga}_{\mathrm{i}}-\mathrm{A}$ & $\mathrm{Ga}_{\mathrm{i}}-\mathrm{B}$ & $\mathrm{Ga}_{\mathrm{i}}-\mathrm{C}$ \\
\hline $\mathrm{S}$ & $1 / 2$ & $1 / 2$ & $1 / 2$ & $1 / 2$ & $1 / 2$ & $1 / 2$ & $1 / 2$ & $1 / 2$ & $1 / 2$ \\
\hline I & $\ldots$ & $\ldots$ & $\ldots$ & $3 / 2$ & $3 / 2$ & $\ldots$ & $3 / 2$ & $3 / 2$ & $3 / 2$ \\
\hline G & $2.00 \pm 0.01$ & $1.96 \pm 0.01$ & $2.01 \pm 0.01$ & $2.00 \pm 0.01$ & $2.00 \pm 0.01$ & $2.04 \pm 0.01$ & $2.00 \pm 0.01$ & $1.99 \pm 0.01$ & $2.00 \pm 0.01$ \\
\hline $\mathrm{A}\left({ }^{69} \mathrm{Ga}\right)\left(\times 10^{-4} \mathrm{~cm}^{-1}\right)$ & $\ldots$ & $\ldots$ & $\ldots$ & $1150 \pm 50$ & $620 \pm 30$ & $\ldots$ & $740 \pm 40$ & $1250 \pm 60$ & $620 \pm 30$ \\
\hline Linewidth (mT) & $60 \pm 6$ & $60 \pm 6$ & $20 \pm 2$ & $20 \pm 2$ & $35 \pm 4$ & $50 \pm 5$ & $35 \pm 4$ & $25 \pm 3$ & $35 \pm 4$ \\
\hline
\end{tabular}

${ }^{\mathrm{a}}$ Only observed after post-growth hydrogen treatment. 


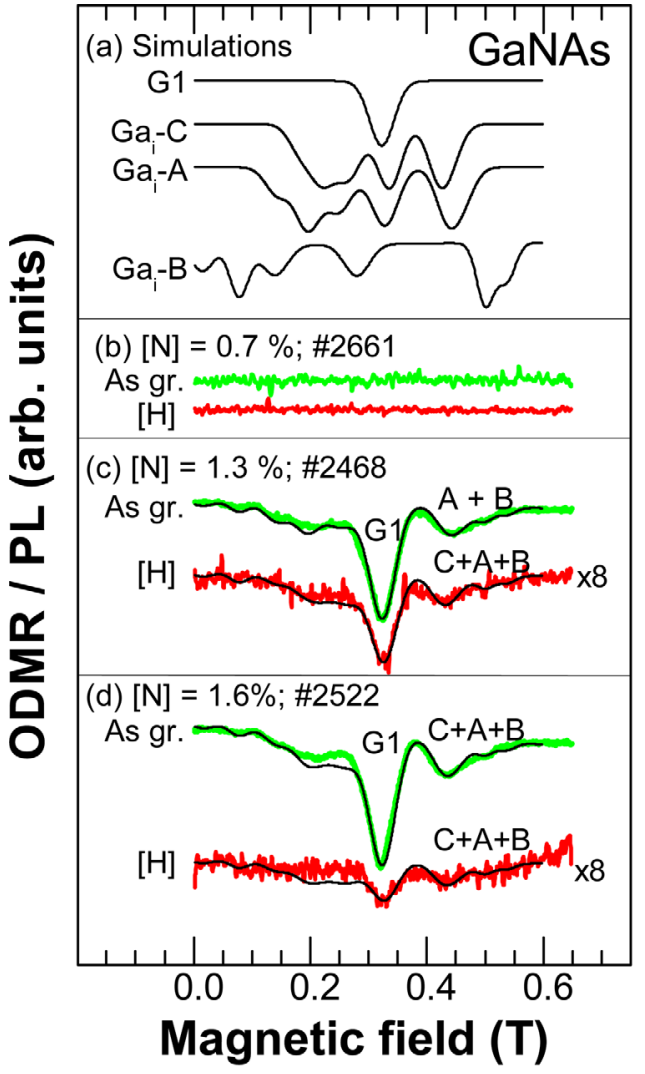

FIG. 5. (Color online) ODMR spectra from GaNAs structures detected at $5 \mathrm{~K}$ by monitoring the near band edge emissions. The ODMR signals are isotropic and are normalized to the PL intensity. Simulated ODMR signals are displayed in (a) whereas the experimentally measured spectra are shown in (b)-(d) for the specified $\mathrm{N}$ compositions. The simulated ODMR spectra are also shown in (b)-(d) by the thin black lines. The spin Hamiltonian parameters used in the simulated spectra are given in Table II.

of the near-band-edge emission caused by combined effects of the H-induced band-gap re-opening and suppression of alloy fluctuations. ${ }^{14,15}$ In addition, a decrease in intensities of the defect-related PL band was observed, likely due to the hydrogen-induced passivation of the corresponding radiative centers. The ODMR spectra from the as-grown samples were found to be very similar when recorded via either the nearband-edge or the defect-related emissions. They contain a number of lines related to several ODMR signals. As in the case of GaNP, the revealed ODMR signals have a negative sign, which evidences that the involved defects act as efficient recombination channels competing with the radiative recombination. However, ODMR signals were suppressed in the hydrogenated structures.

Spin Hamiltonian parameters of the defects were obtained based on an analysis of the measured ODMR spectra by using the spin Hamiltonian given in Eq. (1) and are summarized in Table II. The spectra are found to contain a single Gaussian line signal (denoted as G1 in Fig. 5) from a paramagnetic center $(\mathrm{S}=1 / 2)$ of unknown origin, as well as multi-line signals from several $\mathrm{Ga}_{\mathrm{i}}$-related defects. The latter were identified based on the resolved hyperfine structure and are labeled as $\mathrm{Ga}_{\mathrm{i}}-\mathrm{A}$, $\mathrm{Ga}_{\mathrm{i}}-\mathrm{B}$, and $\mathrm{Ga}_{\mathrm{i}}-\mathrm{C}^{20,36}$ We underline that the same labels are given for the $\mathrm{Ga}_{\mathrm{i}}$-related interstitial complexes in GaNP and GaNAs alloys due to the similarity in their hyperfine interaction

\section{Photon wavelength $(\mu \mathrm{m})$} $\begin{array}{lll}1.5 & 1.2 & 0.9\end{array}$

(a) $[\mathrm{N}]=0.7 \%$; $\# 2661$ GaNAs

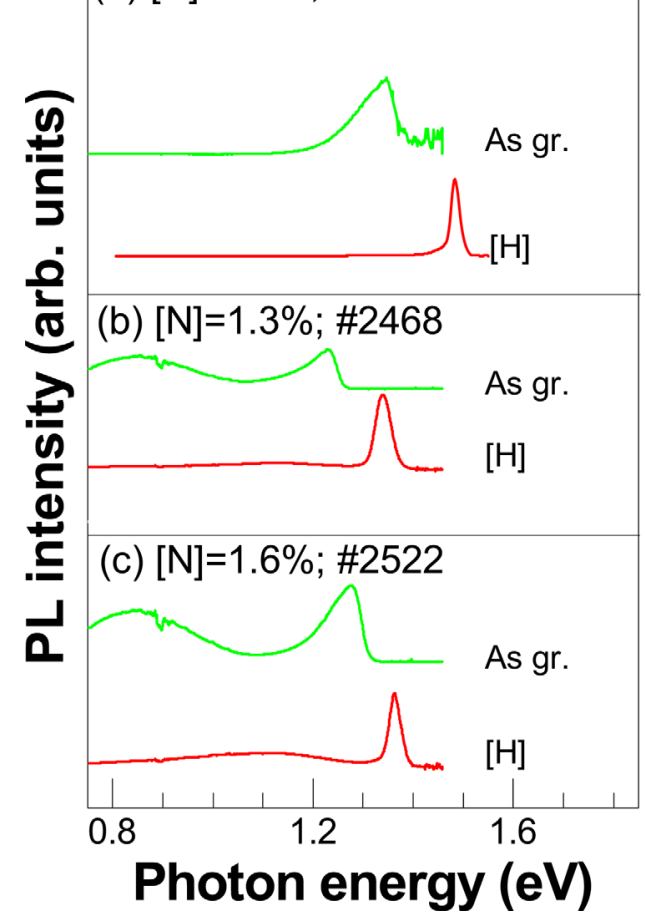

FIG. 6. (Color online) Typical PL spectra measured at $5 \mathrm{~K}$ before and after post growth hydrogenation from (a) a GaNAs epilayer with $[\mathrm{N}]=0.7 \%$, (b) a GaNAs epilayer with $[\mathrm{N}]=1.3 \%$ and (c) GaNAs/GaAs MQW with $[\mathrm{N}]=1.6 \%$. A blueshift of the near-band-edge PL is observed for the GaNAs MQW structure with the highest $\mathrm{N}$ content of $1.6 \%$ as compared with the epilayer sample with $[\mathrm{N}]=1.3 \%$ and is due to the quantum confinement effect.

strengths. The local surrounding of the defects, however, may differ between these materials.

From Fig. 5, the effects of hydrogen incorporation in GaNAs are distinctly different from that observed in the GaNP alloys. First of all, we notice that the main effect of hydrogenation here is dramatic quenching of the ODMR signals that implies efficient passivation of the $\mathrm{Ga}_{\mathrm{i}}$-related defects by H. This behavior is more "traditional," as hydrogen is known to effectively passivate various point defects in semiconductor materials. On the other hand, defect activation if any could only be observed for the GaNAs alloy with $[\mathrm{N}]=1.3 \%$, see Fig. 5(c), where a relative contribution of $\mathrm{Ga}_{i}-\mathrm{C}$ in the measured ODMR spectrum increases in the hydrogenated material. However, since this increase is accompanied with an overall strong quenching of the ODMR intensity, it is hard to reliably conclude whether $\mathrm{Ga}_{\mathrm{i}}-\mathrm{C}$ was indeed activated by $\mathrm{H}$ or it just appears to be stronger because of the quenching of the $\mathrm{Ga}_{\mathrm{i}}-\mathrm{A}, \mathrm{Ga}_{\mathrm{i}}-\mathrm{B}$ signals upon hydrogenation. In turn, this also raises the question whether the $\mathrm{Ga}_{\mathrm{i}}-\mathrm{C}$ complex in GaNAs has the same structure as in GaNP alloys, i.e., if it contains hydrogen as a partner, or the observed similarity in the hyperfine interaction strength for this defect in both materials is purely a coincidence. In the case when $\mathrm{Ga}_{\mathrm{i}}-\mathrm{C}$ was readily formed in the as-grown sample its intensity is found to be reduced by the hydrogenation (Fig. 5(d)). 
The results presented so far clearly show that $\mathrm{H}$ has apparently different effects on the $\mathrm{Ga}_{\mathrm{i}}$-related defects in GaNP and GaNAs alloys. Though the exact physical mechanism for this difference is not currently fully understood and requires future theoretical studies, we may speculate that a possible reason could be a difference in the bandgap energies of these materials. It is possible that in both materials $\mathrm{H}$ interacts similarly with $\mathrm{Ga}_{\mathrm{i}}$ complexes, but the energy level positions of the formed defects relative to the band edges are significantly different. For example, because of the smaller bandgap of GaNAs as compared with that of GaNP, the defect energy level of the formed complex in GaNAs may lie close to either valence or conduction band edge (or even outside the bandgap). This would strongly reduce the role of the corresponding center in carrier recombination making it undetectable by the ODMR technique.

\section{SUMMARY}

In summary, we have employed photoluminescence and optically detected magnetic resonance techniques to investigate effects of post-growth hydrogenation by low-energy sub-threshold ion beam irradiation on defect formation in GaNP and GaNAs alloys. It is found that in GaNP, $\mathrm{H}$ incorporation leads to activation of a new defect which has a $\mathrm{Ga}_{\mathrm{i}}$ atom at its core and may also involve a $\mathrm{H}$ atom as a partner. The observed activation critically depends on the presence of $\mathrm{N}$ in the alloy, as it does not occur in $\mathrm{GaP}$ with a low level of $\mathrm{N}$ doping. In sharp contrast, hydrogen in GaNAs is found to efficiently passivate $\mathrm{Ga}_{\mathrm{i}}$-related defects readily present in the as-grown material. The observed apparent disparity in the $\mathrm{H}$ behavior between GaNP and GaNAs is tentatively ascribed to a difference in the importance of the created $\mathrm{H}$-related defects in carrier recombination due to a difference in their energy level positions with respect to the band edges.

\section{ACKNOWLEDGMENTS}

Financial support by the Swedish Research Council (Grant No. 621-2010-3815) and the Swedish Institute is greatly appreciated.

${ }^{1}$ J. Neugebauer and Chris G. Van de Walle, "Semiconductors and Semimetals," in Hydrogen in Semiconductors II, edited by N. H. Nickel (Academic Press, Boston, 1999), Vol. 61.

${ }^{2}$ C. G. Van de Walle and J. Neugebauer, Annu. Rev. Mater. Res. 36, 179 (2006).

${ }^{3}$ C. T. Sah, J. Y. C. Sun, and J. J. Tzou, Appl. Phys. Lett. 43, 204 (1983).

${ }^{4}$ J. I. Pankove, D. E. Carlson, J. E. Berkeyheiser, and R. O. Wance, Phys. Rev. Lett. 51, 2224 (1983).

${ }^{5}$ N. M. Johnson, C. Herring, and D. J. Chadi, Phys. Rev. Lett. 56, 769 (1986).

${ }^{6}$ W. M. Chen, I. A. Buyanova, A. Buyanov, T. Lundström, W. G. Bi, and C. W. Tu, Phys. Rev. Lett. 77, 2734 (1996)

${ }^{7}$ I. A. Buyanova, W. M. Chen, G. Pozina, W.-X. Ni, G. V. Hansson, and B. Monemar, Appl. Phys. Lett. 71, 3673 (1997).

${ }^{8}$ A. Buyanova, A. C. Ferreira, P. O. Holtz, B. Monemar, K. Campman, J. L. Merz, and A. C. Gossard, Appl. Phys. Lett. 68, 1365 (1996).

${ }^{9}$ P. Caplan, E. Poindexter, B. Deal, and R. Razouk, J. Appl. Phys. 50, 5847 (1979).
${ }^{10}$ See, e.g., A. Amore Bonapasta and M. Capizzi, Defect Diffus. Forum 157-159, 133 (1998), and references therein.

${ }^{11}$ See, e.g., E. E. Haller, W. L. Hansen, and F. S. Goulding, Adv. Phys. 30, 93 (1981), and references therein.

${ }^{12}$ For a review, see Physics and Applications of Dilute Nitrides, edited by I. A. Buyanova and W. M. Chen (Taylor \& Francis, New York, 2004); Dilute Nitride Semiconductors, edited by M. Henini (Elsevier, Oxford, UK, 2005);Dilute III-V Nitride Semiconductors and Material Systems, edited by A. Erol (Springer, Berlin, Germany, 2008).

${ }^{13}$ S. Kleekajai, F. Jiang, K. Colon, M. Stavola, W. B. Fowler, K. R. Martin, A. Polimeni, M. Capizzi, Y. G. Hong, H. P. Xin, C. W. Tu, G. Bais, S. Rubini, and F. Martelli, Phys. Rev. B 77, 085213 (2008).

${ }^{14}$ A. Polimeni, G. Baldassarri H. v., H. M. Bissiri, M. Capizzi, M. Geddo, M. Fischer, M. Reinhardt, and A. Forchel, Phys. Rev. B 63, 201304(R) (2001).

${ }^{15}$ I. A. Buyanova, M. Izafardi, W. M. Chen, A. Polimeni, M. Capizzi, H. P. Xin, and C. W. Tu, Appl. Phys. Lett. 82, 3662 (2003).

${ }^{16}$ A. Polimeni, M. Bissiri, M. Felici, M. Capizzi, I. A. Buyanova, W. M. Chen, H. P. Xin, and C. W. Tu, Phys. Rev. B 67, 201303(R) (2003).

${ }^{17}$ I. A. Buyanova, M. Izadifard, I. G. Ivanov, J. Birch, W. M. Chen, M. Felici, A. Polimeni, M. Capizzi, Y. G. Hong, H. P. Xin, and C. W. Tu, Phys. Rev. B 70, 245215 (2004).

${ }^{18}$ For a comprehensive review, see e.g. A. Polimeni and M. Capizzi, in Physics and Applications of Dilute Nitrides, edited by I. A. Buyanova and W. M. Chen (Taylor \& Francis, New York, 2004), pp. 162-194, and references therein.

${ }^{19}$ I. A. Buyanova, W. M. Chen, and C. W. Tu, Solid State Electron. 47, 467 (2003).

${ }^{20}$ X. J. Wang, Y. Puttisong, C. W. Tu, Aaron J. Ptak, V. K. Kalevich, A. Yu. Egorov, L. Geelhaar, H. Riechert, W. M. Chen, and I. A. Buyanova, Appl. Phys. Lett. 95, 241904 (2009).

${ }^{21}$ H.P. Xin, C. W. Tu, and M. Geva, Appl. Phys. Lett. 75, 1416 (1999).

${ }^{22}$ S. Kurtz, J. Webb, L. Gedvilas, D. Friedman, J. Geisz, J. Olson, R. King, D. Joslin, and N. Karam, Appl. Phys. Lett. 78, 748 (2001).

${ }^{23}$ A. Janotti, S.-H. Wei, S. B. Zhang, Sarah Kurtz, and C. G. Van de Walle, Phys. Rev. B 67, 161201 (2003).

${ }^{24}$ D. Dagnelund, X. J. Wang, C. W. Tu, A. Polimeni, M. Capizzi, W. M. Chen, and I. A. Buyanova, Appl. Phys. Lett. 98, 141920 (2011).

${ }^{25}$ I. A. Buyanova, W. M. Chen, M. Izadifard, S. J. Pearton, C. Bihler, M. S. Brandt, Y. G. Hong, and C. W. Tu Appl. Phys. Lett. 90, 021920 (2007).

${ }^{26}$ For a review, see, e.g., S. J. Pearton, Int. J. Mod. Phys. B 8, 1247 (1994), and references therein.

${ }^{27}$ Marina Berti, Gabriele Bisognin, Davide De Salvador, Enrico Napolitani, Silvia Vangelista, Antonio Polimeni, Mario Capizzi, Federico Boscherini, Gianluca Ciatto, Silvia Rubini, Faustino Martelli, and Alfonso Franciosi, Phys. Rev. B 76, 205323 (2007)

${ }^{28}$ R. Trotta, D. Giubertoni, A. Polimeni, M. Bersani, M. Capizzi, F. Martelli, S. Rubini, G. Bisognin, and M. Berti, Phys. Rev. B 80, 195206 (2009).

${ }^{29}$ I. A. Buyanova, G. Yu. Rudko, W. M. Chen, H. P. Xin, and C. W. Tu, Appl. Phys. Lett. 80, 1740 (2002).

${ }^{30}$ W. M. Chen, Thin Solid Films 364, 45 (2000)

${ }^{31}$ T. A. Kennedy and M. G. Spencer, Phys. Rev. B 57, 2690 (1986).

${ }^{32}$ N. Q. Thinh, I. P. Vorona, I. A. Buyanova, W. M. Chen, S. Limpijumnong, S. B. Zhang, Y. G. Hong, H. P. Xin, C. W. Tu, A. Utsumi, Y. Furukawa, S. Moon, A. Wakahara, and H. Yonezu, Phys. Rev. B 71, 125209 (2005).

${ }^{33}$ The weaker intensities of the experimental ODMR lines at low fields as compared with that in the simulated spectra are because modifications of recombination rates by mixing of states were not included in the simulations, which become more important at the low fields.

${ }^{34}$ V. S. Vavilov, A. E. Kiv, and O. R. Niyazova, Phys. Status Solidi A 32, 11 (1975).

${ }^{35}$ I. A. Buyanova, W. M. Chen, G. Pozina, J. P. Bergman, B. Monemar, H. P. Xin, and C. W. Tu, Appl. Phys. Lett.75, 501 (1999).

${ }^{36}$ I. P. Vorona, T. Mchedlidze, D. Dagnelund, I. A. Buyanova, W. M. Chen, and K. Köhler, Phys. Rev. B 73, 125204 (2006). 